\title{
Hierarchical Approach for Fatigue Cracking Performance Evaluation in Asphalt Pavements
}

\author{
Ibrahim Onifade $^{1^{*}}$, Yared Dinegdae ${ }^{2}$, and Björn Birgisson ${ }^{3}$ \\ ${ }^{1 *}$ Department of Civil and Architectural Engineering, KTH - Royal Institute of Technology, Stockholm, Sweden. E-mail: \\ onifade@kth.se \\ ${ }^{2}$ Department of Civil and Architectural Engineering, KTH - Royal Institute of Technology, Stockholm, Sweden. E-mail: \\ dinegdae@kth.se \\ ${ }^{3}$ Executive Dean, School of Engineering and Applied Science, Aston University, Aston Triangle, Birmingham B4 7ET, United \\ Kingdom. Email: bjorn.birgisson@aston.ac.uk
}

\begin{abstract}
In this paper, a hierarchical approach is proposed for the evaluation of fatigue cracking in asphalt concrete pavements considering three different levels of complexities in the representation of the material behavior, design parameters characterization and the determination of the pavement response as well as damage computation. Based on the developed hierarchical approach, three damage computation levels are identified and proposed. The levels of fatigue damage analysis provides pavement engineers a variety of tools that can be used for pavement analysis depending on the availability of data, required level of prediction accuracy and computational power at their disposal. The hierarchical approach also provides a systematic approach for the understanding of the fundamental mechanism of pavement deterioration, the elimination of the empiricism associated with pavement design and the transition towards the use of sound principles of mechanics in pavement analysis and design.

Keywords: Fatigue cracking; damage; crack initiation; mechanistic approach; pavement analysis.
\end{abstract}

\section{Introduction}

Fatigue cracking is one of the major types of distress in asphalt concrete pavement structure. Fatigue cracking in asphalt pavements is mainly as a result of repetitive application of traffic load on the pavement over a period of time. Fatigue cracking is manifested in asphalt pavements by the presence of randomly distributed or longitudinal crack on the surface of the pavement. Load-induced bending due to application of traffic load is the primary mechanism responsible for the development of fatigue cracking in asphalt pavements. The structure of the pavement layers also plays a significant role in the transfer of traffic load from the surface of the pavement to the underlying layers. For thin pavement layers with weak base layer material, fatigue cracking usually initiate at the bottom of the pavement and propagates to the top, while for thick pavement layers, the crack usually initiate at the top due to tensile stress accumulation at the surface of the pavement in addition to outward shear stresses developed due to the pavement-tire interaction. Other secondary factors that influence the mechanisms of fatigue crack development in asphalt pavements include, environmental effect, subgrade/unbound granular material condition, moisture condition of the pavement structure, hardening of 
the asphalt concrete material with time due to ageing of the binder, healing of the asphalt material, amongst others. The mechanisms of fatigue cracking in asphalt concrete can be classified into different but inter-dependent stages; the micro-crack initiation, micro-crack propagation, coalescence of micro-cracks to form a macro-crack and then eventual failure.

Pavement performance prediction tools with reasonable fatigue cracking prediction accuracy helps to manage cost, presents pavement engineers and managers choices on the strategies for construction and rehabilitation of pavements with primary focus on the prevention of rapid deterioration of the pavement conditions and premature pavement failure. Research efforts to develop tools and models for the prediction of fatigue cracking in asphalt pavements resulted in the development of the mechanistic-empirical pavement design guide (MEPDG) which replaced the American Association of State Highway and Transportation Officials (AASHTO) design procedure due to the inability of the AASHTO method to incorporate significant material properties into the design procedure (ARA Inc., 2004). Asides from the fact that the MEPDG considers the variation in the material properties due to the seasonal weather/climate variations, the MEPDG also utilizes mechanics approach to obtain critical pavement response for the prediction and evaluation of different pavement distress modes such as fatigue cracking and rutting. The MEPDG provides an excellent platform for the further development and integration of more rigorous mechanics-based approach in the design and analysis of flexible pavements. However, the traditional approach for the determination of the critical or terminal condition for fatigue failure used in the MEPDG is based on the Miner's rule, which is used to determine the number of cycles to failure using empirical relationships to relate the strain response to the observed distress in the pavement without considering any fundamental measure of the material's resistance to damage and fracture.

A lot of research effort today are targeted towards the development of mechanistic pavement analysis design procedures and models to eliminate the empiricism associated with existing pavement analysis and design tools. Mechanics-based models rely on the fundamental principles of mechanics to describe the behaviour of asphalt under varying load and environmental conditions. These mechanics-based models consider fundamental material properties such as energy-thresholds to define the resistance of asphalt materials to the development and evolution of damage. For instance, (Roque et al., 1999) identified the existence of fundamental crack growth thresholds below which only healable micro-damage develops and above which non-healable crack initiation or growth occurs in asphalt mixtures. The viscoelastic continuum damage based on the principle of work potential (Schapery, 1990) have been used to characterize damage in asphalt mixtures by numerous researchers e.g., (Kim et al., 2002; Lee et al., 2000; Lee and Kim, 1998; Sullivan, 2008). (Darabi et al., 2012, 2011) proposed a three dimensional constitutive model for nonlinear response and healing of asphaltic materials. (Kim and Little, 1990) developed a one-dimensional constitutive model of asphalt concrete under repetitive load conditions. (Onifade et al., 2015a) developed an energy-based viscoelastic damage and fracture model for modeling of asphalt concrete mixture performance with criteria for micro-crack initiation, propagation and macro-crack formation. (Dinegdae et al., 2015) extended and implemented the Hot Mix Asphalt Fracture Mechanics (HMA-FM) in a pavement analysis framework for prediction of top-down cracking in asphalt pavements. These models consider the mechanism of the strength and degradation of asphalt concrete mixtures in a manner that allows the consideration of complex phenomena such as healing which are non-existent in existing Mechanistic-Empirical (ME) pavement design guide.

In addition to the complexities in modeling the behaviour of asphalt materials, the analysis procedure used for the pavement response prediction, the class of material behaviour used to model the pavement layer materials and damage computation model used for the pavement 
analysis influence the accuracy of the fatigue crack prediction. It has also been shown by various researchers that healing of the induced damage influences the fatigue life of asphalt materials e.g., (Kim et al., 2003; Lee and Kim, 1998; Lytton et al., 2001).

This paper presents a hierarchical approach to the modeling of fatigue cracking in asphalt concrete pavements. The hierarchical approach considers and takes into account different level of complexities in the representation of the material behaviour, design parameters characterization, pavement response prediction and damage computation. The hierarchical approach has been used in the Mechanistic-Empirical (ME) pavement design guide for material and traffic input characterization. However, this paper extends the hierarchical approach to account for the level of complexities in the pavement response analysis and damage computation. The approach presented in this paper helps to provide pavement engineers with a choice of tools and models for fatigue damage prediction depending on the significance of the project, the required level of accuracy of the pavement fatigue performance prediction, availability of required data and computational power. Three levels of damage characterization are identified, Level 3 is traditional fatigue cracking approach using the Miner's rule, Level 2 is based on the dissipated creep strain energy concept using the HMA FM and Level 1 is based on a viscoelastic energybased damage and fracture model. Level 3 gives the least reliable fatigue cracking performance prediction while the Level 1 can be used for more rigorous analysis to obtain higher level of accuracy.

\section{Inputs module}

The Input Module provides all the information that is required for the evaluation of flexible pavements fatigue cracking performance. A hierarchical approach of input parameters characterization is proposed which depends on the level of accuracy required and availability of design input parameters. The main inputs which are required for the evaluation of asphalt pavements fatigue cracking performance are material properties, traffic characterization and climatic condition inputs.

\subsection{Material input characterization}

Material properties are needed to describe the constitutive behaviour of asphalt mixture and the materials that make-up the underlying layers i.e. unbound granular materials and subgrade soil materials. Three hierarchical levels are defined at the material property level for the characterization of the pavement material properties. A similar approach has been used in the MEPDG design guide (ARA Inc., 2004). Level 1 material properties are obtained from elaborate laboratory testing of pavement materials to obtain their mechanical and temperature-dependent properties. Level 1 material characterization provides the most accurate and reliable material property for pavement response simulation. Level 2 material properties can be obtained from predictive models using relationships between constituent material composition and material properties. Level 3 utilizes typical average values from design catalogues or regions without any material testing.

In general, material properties required for asphalt pavement modeling include the viscoelastic (dynamic modulus) and viscoplastic properties, fracture and damage properties, healing and ageing properties as well as the dependency of these properties on temperature. In the case where elaborate experimental testing of the asphalt mixtures is not feasible, predictive models can be used for a Level-2 characterization of the asphalt material properties. These 
predictive models should capture the evolution in materials properties while taking into account effects of aging and healing. For example, in the mechanics-based analysis and design framework by (Dinegdae et al., 2015), the effect of the mixture morphology is incorporated to predict key mixture properties such as the Dissipated Creep Strain Energy (DCSE) and the mixture healing potential. The required inputs for the material property predictive models are the full gradation and volumetric of the mixture, and the performance grade (PG-grade) of the binder.

\subsubsection{Asphalt stiffness aging model}

A calibrated sinusoidal master curve on the basis of the Witczak dynamic modulus equation can be a good alternative for a first level input. The global stiffness aging model can be used to characterize the aged and unaged dynamic modulus of asphalt mixture. The unaged dynamic modulus $\left|E^{*}\right|_{o}$ is predicted based on the Witczak dynamic modulus equation. The change in viscosity of the binder is used to account for the effect of ageing on the asphalt mixture stiffness properties. The global stiffness ageing model is presented in Equation 1:

$$
\left|E^{*}\right|_{t}=\left|E^{*}\right|_{0} \frac{\log \eta_{t}}{\log \eta_{o}}
$$

where $\left|E^{*}\right|_{t}$ and $\left|E^{*}\right|_{o}$ are the dynamic modulus at aged and unaged conditions, and $\eta_{t}$ and $\eta_{o}$ are the viscosity at aged and unaged conditions.

\subsubsection{Dissipated creep strain energy limit model}

The Dissipated creep strain energy (DCSE) is the energy limit or threshold which governs the mixture's resistance against fracture. The DCSE can be obtained from the Superpave IDT strength test by deducing the Elastic Energy (EE) from the Fracture Energy (FE) density of the material. The DCSE can be predicted using morphology-based predictive material model which developed based on the relationship between the experimentally determined $D C S E_{\text {lim }}$ of 49 asphalt mixtures with their morphological configuration and age. The Primary Structure (PS) coating thickness $\left(t_{p}\right)$ is the morphology parameter used in establishing the relationship. Details about how to obtain the morphology parameter can be found in (Das et al., 2013; Lira et al., 2012; Onifade et al., 2015b). The DCSE predictive equation is presented in Equation 2

$$
D C S E_{\text {lim }}=k_{1}\left(\frac{1}{t_{p}}\right)^{k_{2}}\left(\frac{1}{t}\right)^{\left(k_{3}+k_{4} \log \left(t_{p}\right)\right)}
$$

where $k_{1}, k_{2}, k_{3}$ and $k_{4}$ are model constants and evaluated as $2.38,0.79,0.33$ and 0.12 respectively. Though not excellent for the whole range of PS coating thickness, the above equation has been shown to deliver reasonable $D C S E_{\text {lim }}$ predictions for mixtures with PS coating thickness values between 0.75 and $1.25 \mathrm{~mm}$.

\subsubsection{Asphalt Creep model}

The asphalt concrete creep model is used to predict the creep response of asphalt material as a function of temperature and applied load. The creep compliance parameters obtained from experimental testing of the material can be used to determine the creep strain of a given mixture at any time. 


\subsection{Traffic}

Traffic related inputs are required for pavement response prediction and damage computation. A three level hierarchical approach is proposed for traffic characterization similar to the procedure in MEPDG.

Level 3 traffic input utilizes the Equivalent Single Axle Load (ESAL) for the traffic characterization. Level 2 inputs are characterized based on axle load spectra which are obtained by analysing traffic data from project specific weigh-in-motion (WIM) stations or from a regionwide WIM default values. Level 1 traffic characterization uses the traffic load spectra with all its associated inputs such as vehicle class distribution, seasonal traffic variation, traffic growth rate and lateral wheel wander. The Level 1 provides the most accurate and reliable data for pavement performance prediction.

\subsection{Climate input model}

Climate inputs play a critical role in the resultant performance of asphalt concrete flexible pavements. Effect of varying climatic condition on the pavement temperature and moisture in unbound granular layers have a direct influence of layers modulus which reflects in the pavement response and pavement performance. A two level hierarchical approach for climate related inputs were also proposed that takes into consideration the availability of climate related parameters which are necessary to predict the climate inputs.

Two different models are identified for the prediction of the variation in the climatic conditions, i.e. simplified climate prediction models and the Enhanced Integrated Climatic Model (EICM). The temperature predictive model developed by (Viljoen, 2001) to estimate the temperature levels at different depths in the AC layer and the moisture distribution model developed by (Yideti et al., 2014) are simplistic climate prediction models that can used to predict the variation in temperature and moisture conditions of bound and unbound granular materials. The EICM is an advanced climate prediction models which have been calibrated and validated using extensive data. The EICM is the climate model used in the MEPDG.

The climate models are used to predict variation in properties such as the Mean Annual Air Temperature (MAAT), pavement temperature gradient in the AC layer, moisture conditions in the unbound layers, capillary suction level, ground water level and frost penetration level.

\section{Pavement Response prediction}

The pavement response model computes the field variables (stress, strain and deformation) in the pavement structure as a result of the application of traffic load and varying environmental conditions. The computed responses are then inputted into the pavement damage computation model for the prediction of pavement distress e.g., fatigue cracking.

The pavement response model considers the periodic variation in the temperature of the AC mixture across the AC layer thickness through the climatic model and the effect of temperature gradient across the AC layer is reflected on the predicted AC layer stiffness. Different classes of materials and different types of analyses can be setup to compute the pavement response depending on the available data, accuracy of the prediction, and the computational capacity.

The Multi-layered Elastic Analysis (MLEA) and the Finite Element Analysis (FEA) are two types of analyses used for analyzing pavement structures. The MLEA assumes an axisymmetric geometry with linear elastic material behaviour for all materials in the pavement structure using the elastic material properties (e.g., Young's modulus and Poisson's ratio) to 
model the pavement structural behaviour. The effect of material non-linearity (such as the effect of the stress-state in the Unbound granular materials on the Resilient Modulus) cannot be incorporated directly into the constitutive behaviour of the pavement materials. The LEA provides a rather quick and approximate measure of the pavement response under prescribed load conditions.

The FEA on the other hand provides a more rigorous analysis framework for pavement structural response computation. The FEA presents the possibility to take into account, the effects of non-linearity on the pavement response (such as the effect of stress-states and moisture on unbound granular material stiffness), viscoelastic and viscoplastic behaviour of the asphalt concrete mixtures, the effect of damage on the constitutive behaviour of the pavement materials, as well as the effect the load history on the pavement state and condition. The FEA can be used to perform axi-symmetric, 2D and 3D analysis of pavement structures. Axi-symmetric models limit the contact area to a circular shape, while 2D and 3D analysis can take account more realistic load profiles.

\section{Damage computation}

Damage computation is the prime task of any pavement performance and analysis framework. Damage computation models are used to predict the distress (in this case fatigue cracking) in pavement structures as a result of the pavement response computation due to the application of traffic load and varying environmental conditions.

Traditional empirical fatigue models predictions are based on the tensile strain at the bottom of the AC layer and the stiffness of the AC layer material. These empirical models require extensive calibration to match the model prediction to the pavement response due to the sensitivity of the measured strain at the bottom of the AC layer to changes in the pavement structure. The empirical models also do not require any material damage parameter to describe the initiation or evolution of damage. Unlike the traditional empirical models, Mechanics-based models consider the influence of the pavement structure on the pavement structural response and fundamental material properties are established and used as a measure of the material's resistance to damage. The influence of healing and ageing on the fundamental materials damage resistance threshold is considered which results in a more accurate representation of the strength and degradation mechanism in pavement structures. The use of the fundamental underlying mechanics principles in pavement analysis and design will result in material models that doesn't require extensive calibration to relate the model damage prediction and the observed pavement distress.

The following sub-sections presents three different levels of models that can be used for fatigue cracking prediction in asphalt pavements. The traditional Mechanistic-Empirical (ME) pavement design guide fatigue cracking model is designated a Level 3 fatigue model, the mechanics-based fatigue cracking prediction model based on the HMA-FM is designated a level 2 fatigue model, and the energy-based viscoelastic damage and fracture model is typical of a Level 1 model. Figure 1 shows the flow diagram for the proposed hierarchical approach for pavement performance evaluation. The flow diagram can be adapted to the different hierarchical levels giving the user the flexibility in choice of material models and damage computation. 


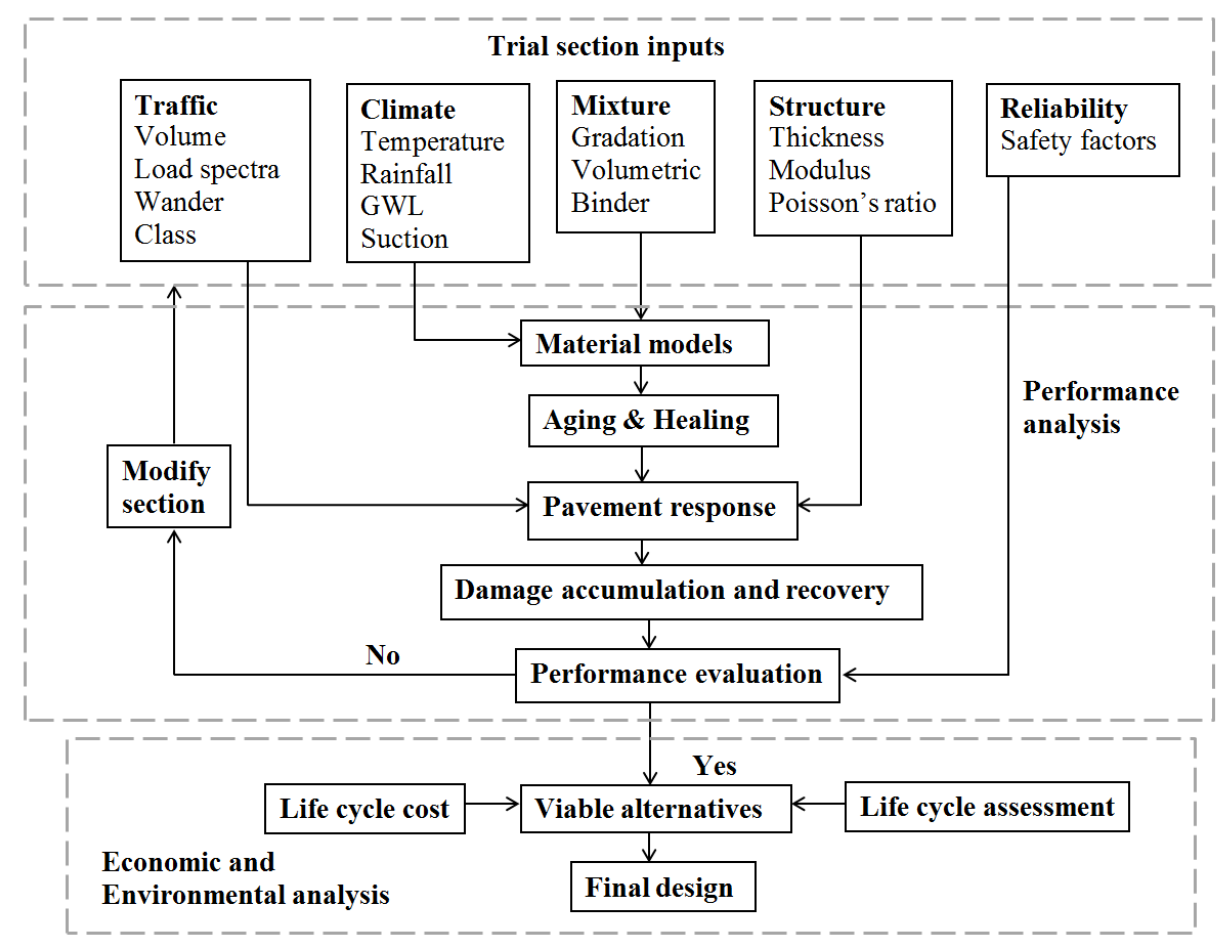

Figure 1: Analysis flow diagram for the hierarchical pavement analysis framework

\subsection{Level 3 - Traditional Fatigue model}

The traditional fatigue model is based on empirical models developed based on the correlation between empirical relationships and observed pavement response. This empirical models doesn't not account for fundamental mechanisms of the crack initiation and propagation, structural composition of the pavement layers and does not account for the effect of damage on the constituent material behaviour. The form of the fatigue cracking model used in the MEPDG is presented in Equation 3.

$$
N_{f}=C \beta_{1} k_{1}\left(\frac{1}{\varepsilon_{t}}\right)^{\beta_{2} k_{2}}\left(\frac{1}{E}\right)^{\beta_{3} k_{3}}
$$

Where:

$N_{f}$ : is the total number of cycles to failure

$\varepsilon_{t}$ : tensile strain at the critical location

$E$ : Stiffness of the material

$k_{1}, k_{2}, k_{3}$ : Laboratory regression coefficients

$\beta_{1}, \beta_{2}, \beta_{3}$ : Calibration parameters

$C$ : Laboratory to field adjustment factor

The final model used for the prediction of the number of repetitions to fatigue cracking after the national field calibration in the US is given as:

$$
N_{f}=0.00432 k_{1}^{\prime} C\left(\frac{1}{\varepsilon_{t}}\right)^{3.9492}\left(\frac{1}{E}\right)^{1.281}
$$


where $\beta_{1}, \beta_{2}, \beta_{3}$ are obtained as $1.0 k_{1}^{\prime}, 1.2$ and 1.5 respectively. $k_{1}^{\prime}$ was introduced as a correction for different asphalt layer thickness.

The laboratory to field adjustment factor $C$ is defined as:

$$
C=10^{M}
$$

where $M$ is defined as a function of the percentage effective binder content $\left(V_{b}\right)$ and the percentage air voids content $\left(V_{a}\right)$ and expresed as:

$$
M=4.84\left(\frac{V_{b}}{V_{a}+V_{b}}-0.69\right)
$$

The asphalt layer thickness correction factor $k_{1}^{\prime}$ for both bottom-up fatigue cracking and top-down fatigue cracking are given as:

$$
\begin{gathered}
k_{1}^{\prime}=\frac{1}{0.000398+\frac{0.003602}{1+\exp ^{11.02-3.49 \cdot H_{a c}}}} \text { bottom-up } \\
k_{1}^{\prime}=\frac{1}{0.01+\frac{12}{1+\exp ^{15.676-2.8186 \cdot H_{a c}}}} \text { top-down }
\end{gathered}
$$

\subsection{Level 2 - mechanics-based crack initiation (CI) prediction framework}

The mechanics-based crack initiation (CI) prediction framework was developed based on the fundamental energy threshold concept HMA-FM (Roque et al., 2002; Zhang et al., 2001). The HMA-FM is based on the concept that there is an energy threshold referred to as the dissipated creep strain energy limit of asphalt mixtures, which, when exceeded by the induced damage, creates a non-healable macro crack. The mechanics-based crack initiation accounts for the effect of healing and degradation in viscoelastic material properties due to ageing during the service life of the pavement.

The stress and strain response from the pavement response analysis is used to compute the damage in the pavement structure due to the applied load. The pavement response can consider both linear and non-linear material behaviour. Equation 9 shows the expression used to compute load associated damage per cycle, where $\sigma$ is the stress from the pavement response computation and $\dot{\varepsilon}_{\text {pmax }}$ is the creep strain rate of the material. Equation 9 shows the damage computation for a haversine load function with a load duration of $0.1 \mathrm{~s}$ and a rest period of $0.9 \mathrm{~s}$. Different load functions can be incorporated into the model for damage computation.

$$
\operatorname{DCSE}_{L} / \text { cycle }=\int_{0.1}^{0} \sigma \cdot \sin (10 \pi t) \cdot \dot{\varepsilon}_{\text {pmax }} \cdot \sin (10 \pi t) \cdot d t
$$

The accumulated damage over the total analysis period $\left(t_{a}\right)$ is computed using Equation 10 where, $\operatorname{ESAL}\left(t_{i}\right)$ is the total Equivalent Standard Axle Load for each analysis period $\left(t_{i}\right)$. In this case, an hourly analysis period is considered for response computation and damage analysis.

$$
\operatorname{DCSE}_{\text {accum }}(t)=\sum_{t_{i}=1}^{t_{a}} \operatorname{ESAL}\left(t_{i}\right) \cdot \operatorname{DCSE} E_{L} / \text { cycle }
$$

Asphalt mixtures have the capacity to heal induced damage when subjected to favourable temperature conditions and allowed enough rest period before application of further load. The 
healing of damage in the asphalt pavements is taken into account through the introduction of a healing potential. The healing potential is established as a function of the morphological parameter $\left(t_{p}\right)$ and the initial Dissipated Creep Strain Energy $\left(D C S E_{i}\right)$ of the mixture. The expression derived for the yearly healing potential is given as:

$$
h_{y m}(t)=1-\left(\left[\exp \left(\frac{t_{p}}{t}\right)^{-D C S E_{i}}\right]_{n o r m}\right)^{k}
$$

where $k$ is a parameter used for the calibration of the model to observed field performance. $k$ is expressed as a function of the morphology of the material using the morphological parameter $\left(t_{p s}\right)$.

The remaining DCSE is obtained after the recovered DCSE due to the healing is subtracted from the total accumulated DCSE. The remaining DCSE is obtained using the expression in Equation 12.

$$
D C S E_{\text {remain }}(t)=D C S E_{\text {accum }}(t) \cdot\left(1-h_{y m}(t)\right)
$$

The critical condition for crack initiation is considered as the state where the remaining accumulated DCSE in the material due to load application is equal to the DCSE limit of the material. At this limit state, a crack initiates in the asphalt pavement which is then propagated by further traffic load application, variations in environmental condition and moisture infiltration into the surface and underlying pavement layers. The limit state condition that signals the crack initiation event is expressed as:

$$
t_{\text {init }}=D C S E_{\text {remain }}(t)-D C S E_{\text {lim }}(t)
$$

where, $t_{\text {init }}$ is the time in years for crack initiation, $D C S E_{\text {remain }}$ is the remaining accumulated $D C S E$ and $D C S E_{\text {lim }}$ is the DCSE limit of the material.

\subsubsection{Mechanics-based model calibration and validation results (level 2)}

For the calibration and validation of the mechanics-based fatigue crack initiation framework, twenty-eight (28) different pavement sections from Florida, which include state roads, turnpikes and interstates were selected for analysis of top-down cracking initiation prediction. The relevant information needed as input in the analysis framework include asphalt mixture gradation and volumetric properties, cross-sectional properties, traffic, temperature, etc. The observed crack initiation time of the pavement sections were obtained from the Florida Department of Transportation database (FloridaDepartmentofTransportation(FDOT), 2013). Sixteen (16) pavement sections were selected for model calibration and categorized into two different groups. Pavement sections with an annual traffic volume of 100,000 ESALS or less are referred to as "low traffic volume group" while those with annual traffic volume of more than 100,000 ESALS are referred to as "high volume group". The "low volume group" and the "high volume group" calibration of the pavement sections resulted in a calibration factors for both traffic volume groups. The calibration factor $(k)$ in Equation 11 for the "low volume group" is $14.09 \cdot t_{p s}+4.39$, while the calibration factor $(k)$ for the "high volume group" is $15.5 \cdot t_{p s}+3.35$.

Figures $2 \mathrm{a}$ and $2 \mathrm{~b}$ show the calibration results for the medium to high volume roads and the low volume roads respectively. Due to the variations in the input factors that influence the long term performance of asphalt pavement sections, a crack initiation time of \pm 3 years from the observed crack initiation time in the field is considered a "good" prediction. For the "medium 


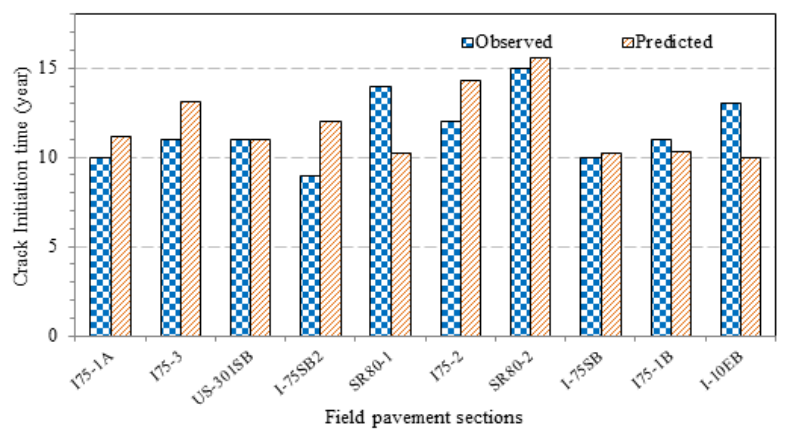

(a) Medium to high volume roads

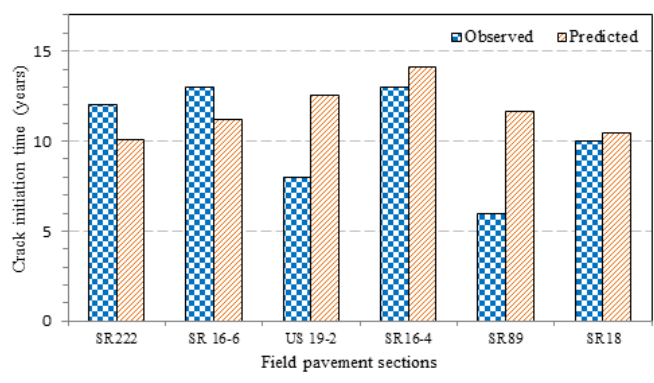

(b) Low volume roads

Figure 2: Predicted and observed crack initiation time (years) for medium to high volume roads and low volume roads

to high volume roads", it can be seen that the predicted crack initiation time is consistent with the observed crack initiation time except for SR80-1 that deviates from the observed value by -3.7 years. For the "low volume roads", the predicted crack initiation time is also in the range of acceptable prediction except for US19-2 and SR89 that deviated by more than 3years. Twelve (12) pavement sections are used for the validation of the model. Figure 3 shows the result of the model validation. The result of the validation shows that the framework is capable of predicting the top-down crack initiation time to a reasonable extent.

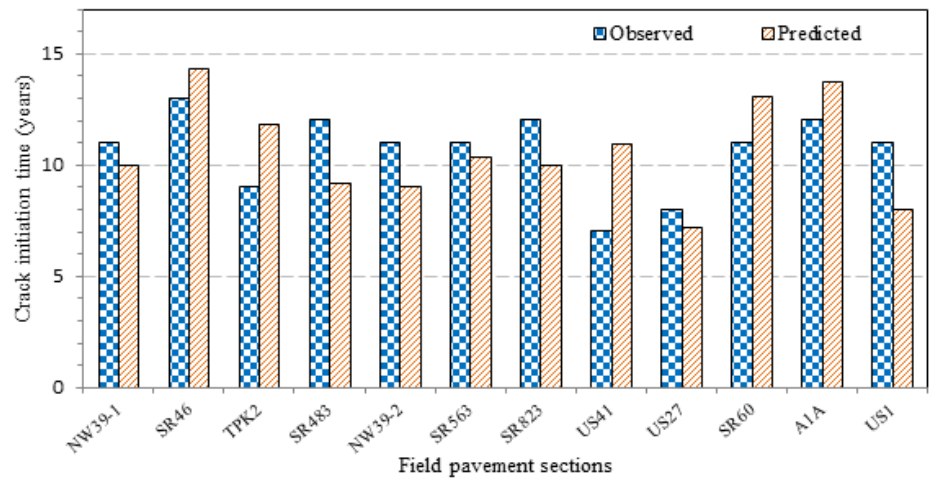

Figure 3: Model validation result using 12 different pavement sections

With the consideration of the influence of the morphology on the key mixture properties, the framework is able to predict the top-down crack initiation time with considerable accuracy. The model has accounted for the fundamental mechanism of pavement degradation on which further study will be based. More accurate traffic characterization will improve the prediction capability of the framework.

\subsection{Level 1 - Energy-based damage and fracture model}

Recently (Onifade et al., 2015a) proposed a new viscoelastic damage model based on energy balance with potentials for the identification of the critical threshold for micro-crack initiation and its consequent evolution based on thermodynamics of irreversible processes and Continuum Damage Mechanics.

The generalized Maxwell model of n-terms with inclusion of a viscous strain $\left(\varepsilon_{i}^{v}\right)$ from each of the dashpot components is used in the model formulation. The generalized Maxwell model 
is categorized into a long term equilibrium (time-independent) part with long term equilibrium stiffness $\left(E_{\infty}\right)$ and a series of non-equilibrium parts (time-dependent) with different stiffness $\left(E_{i}\right)$ and dashpot viscosity $\left(\eta_{i}\right)$. The total strain is additively decomposed into an elastic strain $\left(\varepsilon_{i}^{e}\right)$ and a viscous strain $\left(\varepsilon_{i}^{v}\right)$ on each Maxwell branch i.e. $\varepsilon=\varepsilon_{i}^{e}+\varepsilon_{i}^{v}$.

The viscoelastic damage formulation is based on the energy equivalence principle with anisotropic damage representation to capture the behaviour of asphalt concrete in tension and in compression. The anisotropic damage variable takes the form of a second-order tensor (D) and the integrity tensor $\phi$ is identified and expressed as $\phi=I-\mathbf{D}$, where $I$ is a second-order identity matrix and principal values of both $\phi$ and $\mathbf{D}$ varies from 0 to 1 .

Considering the energy equivalence principle, the effective stress and strain are related to their nominal counterpart and expressed as shown in Equation 14 and Equation 15:

$$
\begin{gathered}
\bar{\sigma}=M: \sigma, \\
\bar{\varepsilon}=M^{-T}: \varepsilon,
\end{gathered}
$$

where $\sigma$ is the nominal stress and $\bar{\sigma}$ is the effective stress, $\varepsilon$ is the nominal strain and $\bar{\varepsilon}$ is the effective strain, and $M$ is a fourth-order damage tensor.

The fourth-order damage tensor $M$ is defined in terms of the integrity tensor $\phi$ (where $\phi=I-\mathbf{D})$ according to (Voyiadjis et al., 2009) as:

$$
M=\frac{1}{2}\left(I \otimes \phi^{-1}+\phi^{-1} \otimes I\right),
$$

The matrix representation of $M^{-1}$ tensor exhibits the diagonal form shown in Equation 17 in the principal axis of damage. A similar representation of the fourth-order damage tensor was used by (Carol et al., 2001), (Abu Al-Rub and Voyiadjis, 2003):

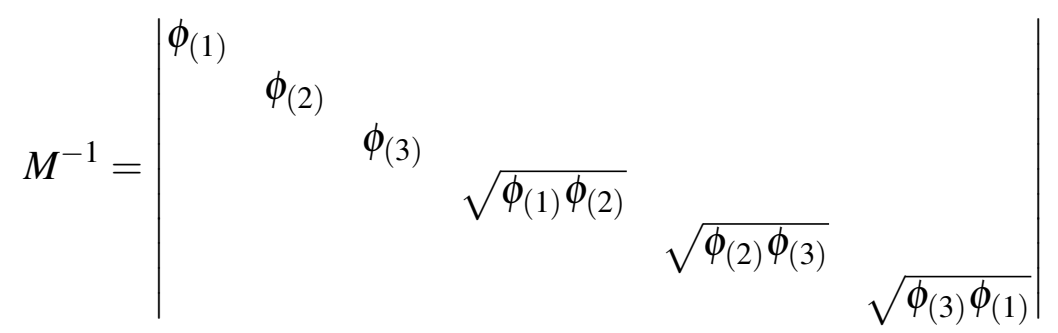

The relationship between the damaged stiffness $E_{\infty}$ and the undamaged (effective) stiffness $\bar{E}_{\infty}$ for the long term part is shown in Equations 18. For the Equilibrium parts, Equation 19 shows the relationship for the damaged stiffness $E_{1}$ and the undamaged (effective) stiffness $\bar{E}_{1}$.

$$
\begin{gathered}
E_{\infty}=M^{-1}: \bar{E}_{\infty}: M^{-T}, \\
E_{i}=M^{-1}: \bar{E}_{i}: M^{-T},
\end{gathered}
$$

The damaged equilibrium term stiffness $E_{i}$ can be written in terms of the effective equilibrum Lame's constants $\bar{\lambda}_{i}$ and $\bar{G}_{i}$ and Equation 18 is expressed as:

$$
E_{i}=M^{-1}: M^{-T}\left(\bar{\lambda}_{i}+2 \bar{G}_{i}\right)
$$

The matrix representation of the stiffness matrix for the equilibrium part is given as: 


$$
E_{i}=\left|\begin{array}{cccccc}
\phi_{(1)}^{2}\left(\lambda_{i}+2 \bar{G}_{i}\right) & \bar{\lambda}_{i} & \bar{\lambda}_{i} & & & \\
\bar{\lambda}_{i} & \phi_{(2)}^{2}\left(\bar{\lambda}_{i}+2 \bar{G}_{i}\right) & \bar{\lambda}_{i} & & & \\
\bar{\lambda}_{i} & \bar{\lambda}_{i} & \phi_{(3)}^{2}\left(\bar{\lambda}_{i}+2 \bar{G}_{i}\right) & & & \\
& & & \phi_{(1)} \phi_{(2)} \bar{G}_{i} & & \\
& & & \phi_{(2)} \phi_{(3)} \bar{G}_{i} & \\
& & & & \phi_{(3)} \phi_{(1)} \bar{G}_{i}
\end{array}\right|
$$

Similar representation used in Equations 20 and Equation 21 are used for the stiffness matrix of the long-term part $E_{\infty}$.

The spectral decomposition technique introduced by (Simo and Ju, 1987a), (Simo and Ju, $1987 \mathrm{~b}$ ) is used to decompose the stress and strain tensors into positive and negative components so as to distinguish between the behaviour of asphalt concrete in tension and compression. For example, the negative component of the stress tensor is obtained by subtracting the positive component $\left(\sigma^{+}\right)$obtained using the spectral decomposition technique from the total stress tensor $(\sigma)$. The negative part of the stress tensor can be obtained using the expression in Equation 22

$$
\sigma^{-}=\sigma-\sigma^{+}
$$

Once the positive and negative components of the stress and strain tensor have been obtained, the damage conjugate $(Y)$ can then be decomposed to obtain the positive $\left(Y^{+}\right)$and negative $\left(Y^{-}\right)$parts as well. $\left(Y^{+}\right)$is the damage driving force under tensile conditions while $\left(Y^{-}\right)$is the driving force under compressive conditions. The damage conjugate $(Y)$ is written in terms of the of the total effective stress $\bar{\sigma}$ and the total viscoelastic strain $\varepsilon$ as:

$$
Y^{ \pm}=\frac{1}{2}\left[\varepsilon^{ \pm} \cdot \bar{\sigma}^{ \pm}+\bar{\sigma}^{ \pm} \cdot \varepsilon^{ \pm}\right]
$$

where \pm designates the positive and the negative components of the stress and strain tensors.

\subsection{Damage initiation and evolution}

A non-associative damage formulation is used to derive different criteria for damage initiation and evolution. The initiation and evolution of damage is considered only on the positive part of damage conjugate $Y^{+}$. The micro-crack initiation criteria is expressed in terms of the micro-crack initiation potential $\left(\wp_{1}^{*}\right)$, the critical micro-crack initiation threshold $\left(\wp_{1, c}^{*}\right)$, and the isotropic hardening parameter. The micro-crack initiation criterion in one principal damage axis is expressed as follows:

$$
f^{d}=\wp_{1}^{*}\left(Y^{+}\right)-\wp_{1, c}^{*}\left(S_{o}\right)-R(r)=0
$$

Where:

$$
\begin{aligned}
& \wp_{1}^{*}\left(Y^{+}\right) \text {: is the micro-crack initiation potential } \\
& \wp_{1, c}^{*}\left(S_{o}\right) \text { : is the critical micro-crack initiation threshold } \\
& Y^{+}: \text {is a measure of the strain energy density } \\
& S_{o}: \text { is an energy term obtained from a strength test }
\end{aligned}
$$


The micro-crack initiation potential $\wp_{1}^{*}\left(Y^{+}\right)$is driven by thermodynamic conjugate of the damage variable $\left(Y^{+}\right)$and expressed as:

$$
\wp_{1}^{*}\left(Y^{ \pm}\right)=\frac{S_{o}}{k_{2}+1} \cdot\left(\frac{\sqrt{Y^{+}: Y^{+}}}{2 \cdot S_{0}}\right)^{k_{2}+1}
$$

where $k_{2}$ and $S_{o}$ are material parameters. The critical micro-crack initiation threshold $\wp_{1, c}^{*}\left(S_{o}\right)$ is expressed in terms of $k_{2}$ and $S_{o}$ as:

$$
\wp_{1, c}^{*}\left(S_{o}\right)=\frac{S_{o}}{k_{2}+1}
$$

The micro-crack propagation criterion $F_{D}$ which is used to derive the evolution of damage is expressed as:

$$
F_{D}=\wp_{2}^{*}\left(Y^{+}\right)-\alpha \cdot \wp_{1, c}^{*}\left(S_{o}\right)-R(r)=0,
$$

where $\alpha$ is $k_{1} / k_{2} \cdot \wp_{2}^{*}\left(Y^{+}\right)$is the micro-crack propagation potential and expressed as:

$$
\wp_{2}^{*}\left(Y^{+}\right)=\alpha \cdot \frac{S_{o}}{k_{2}+1} \cdot\left(\frac{\sqrt{Y^{+}: Y^{+}}}{2 \cdot S_{0}}\right)^{k_{2}+1}
$$

The evolution of micro-crack is obtained with respect to the dissipative micro-crack potential $F_{D}$ by taking the derivative of the dissipation potential. The resulting power-law type damage evolution law is given as:

$$
\dot{D}=\frac{k_{1}}{k_{2}} \cdot\left(\frac{\sqrt{Y^{+}: Y^{+}}}{2 \cdot S_{0}}\right)^{k_{2}} \cdot \frac{Y^{+}}{\sqrt{Y^{+}: Y^{+}}} \cdot \dot{r}
$$

where, $k_{1}, k_{2}$ and $S_{o}$ are material parameters.

\subsubsection{Macro-crack Formation}

The fracture energy obtained by means of differential strain gauge observation in the Superpave IDT strength test has been used as the energy limit for macro-crack initiation. It has been shown in several studies that the fracture energy is a fundamental material property that is independent of stress state and loading conditions which is adequate to evaluate mixture fracture potential e.g., (Koh, 2009; Zhang et al., 2001). The Fracture energy can be predicted using a powerlaw type relationship between the material parameter $k_{1}$ and the mixture fracture energy $(F E)$ density for the conventional mixtures. The relationship between $k_{1}$ and FE for the conventional mixtures is expressed as:

$$
F E=A \cdot k_{1}^{-n}
$$

where $A$ and $n$ are model constants. Typical values of the model constants $A$ and $n$ are 45 and 0.76 respectively for the asphalt mixtures used for the model development. 


\subsubsection{Temperature Coupling}

A temperature coupling model is integrated into the damage model to obtain the critical microcrack initiation threshold, and the material damage parameters $\mathrm{k} 1$ and $\mathrm{k} 2$ over a wide range of temperatures. The temperature coupling model is used to predict the material damage characteristics at temperatures not tested for thereby reducing the amount of experiment needed to describe the material characteristic behaviour over wide temperature ranges which the material is exposed to during their service life. The coupling expressions for the temperature-dependent critical micro-crack initiation threshold $\wp_{1, c}^{*}(T)$, and the material damage parameters $k_{1}(T)$ and $k_{2}(T)$ are presented in Equation 31 - Equation 33:

$$
\begin{gathered}
\wp_{1, c}^{*}(T)=\wp_{1, c . r e f}^{*}(T) \cdot G(T) \\
\log \left(k_{1}(T)\right)=\log \left(k_{1 . r e f}\right) \cdot H(T) \\
k_{2}(T)=\left(k_{2 . r e f}\right) \cdot B(T)
\end{gathered}
$$

where, $G(T), H(T)$, and $B(T)$ are temperature-dependent coupling functions. The expressions for the temperature-coupling functions are given in Equation 34 - Equation 36:

$$
\begin{gathered}
G(T)=\exp -\left(\theta_{1} \cdot \log \left(a_{T}\right)\right) \\
H(T)=\exp -\left(\theta_{2} \cdot \log \left(a_{T}\right)\right) \\
B(T)=\exp \left(-\theta_{3} \cdot\left(1-\frac{T}{T_{r e f}}\right)\right)
\end{gathered}
$$

where $a_{T}$ is the temperature-dependent master curve shift function, and $\theta_{1}, \theta_{2}, \theta_{3}$ are temperature coupling parameters which can be obtained from material strength testing.

\subsubsection{Healing and Ageing}

The coupling of the energy-based damage and fracture model with the temperature enables the easy incorporation of the effects of healing and ageing on the asphalt mixture response. Several publication have supported the potential for asphalt mixtures to heal induced damage when subjected to repeated cyclic loads. The general conclusion in the majority of studies of healing in asphalt mixtures under repeated loading shows that the material can withstand less amount of load repetitions before eventual failure for load profiles without rest period between consecutive load applications. However, when a rest period is introduced, it was observed that the material can withstand more load repetitions before eventual failure e.g., (Daniel and Kim, 2001; Kim et al., 2003; Lee and Kim, 1998; Lytton et al., 2001). Consideration of the healing phenomenon as observed in asphalt mixtures is necessary in mechanics-based pavement performance prediction model for a realistic prediction of the pavement response and distress.

(Little and Bhasin, 2007) proposed that the phenomenological difference between viscoelastic recovery and healing is that while the former is due to the rearrangement of molecules within the bulk of the material, the latter is due to the wetting and inter-diffusion of material between the two faces of a nano crack to achieve properties of the original material. In an effort towards the development and incorporation of healing mechanism for use within the framework of mechanics-based pavement analysis, three different healing phenomena types of healing mechanisms are identified and proposed 
- Load-dependent healing: healing is due to relaxation of stresses which results in the wetting and inter-diffusion of material between two micro-crack surfaces after the removal of load application. Healing process requires adequate rest period between consecutive load applications for healing of induced damage to occur. Load-dependent healing is not possible when an energy threshold has been exceeded and significant amount of damage has been induced in the material. Load-dependent healing is typical of the healing process in the laboratory at constant temperature.

- Temperature-dependent healing: The propensity of wetting and inter-diffusion of materials between two micro-crack surfaces increases as the temperature increase. This implies that healing capacity/potential of a material increases when the temperature of the material is increased. Full recovery and healing of all induced damage is possible as long as the material is subjected to favourable temperature conditions.

- Load and Temperature-dependent healing: This is the combination of the load-dependent and the temperature dependent healing mechanisms. This type of mechanism is typical of what is experienced in real flexible pavements (field conditions).

The energy-based damage and fracture model has the possibility to capture both loaddependent and temperature-dependent healing mechanisms in a thermodynamically consistent manner through the micro-crack propagation potential and the temperature dependent critical micro-crack initiation potential respectively.

\subsubsection{Level 1: Energy-based damage and fracture model - Fatigue crack prediction}

The energy-based viscoelastic damage and fracture model is used for the fatigue analysis of asphalt concrete mixture subjected to cyclic load conditions at $-10^{\circ} \mathrm{C}$. The material property to model the material response are obtained from an earlier study, where the Superpave IDT resilient modulus and creep test are used to obtain the linear viscoelastic response. The material damage parameters $k_{1}, k_{2}$ and $S o$ are obtained from the Superpave IDT strength test. The values of the damage parameters $k_{1}, k_{2}$ and $S o$ are $203,0.7$ and $0.3 \mathrm{~kJ} / \mathrm{m}^{3}$ respectively. The material is subjected to a Haversine pressure functions with three different frequencies of $1 \mathrm{~Hz}, 3 \mathrm{~Hz}$ and $4 \mathrm{~Hz}$. The haversine pressure function can be expresses as:

$$
\sigma=[\sin (n \pi t) \sin (n \pi t)+0.2] \cdot 1000 k P a
$$

where $\sigma$ is the applied pressure, $n$ is the frequency and $t$ is the time. The function in Equation 37 is repeated for every 1 second period. From the function in Equation 37, the applied maximum and minimum pressure are $1200 \mathrm{kPa}$ and $200 \mathrm{kPa}$ respectively. Figure 4a shows the plot of the applied load function when $\mathrm{n}=4$.

The prony series representation of the relaxation spectrum is made up of a 2-leg generalized Maxwell model. The long term stiffness $E o$ is $0.21 G P a$ while the equilibrium stiffness $E 1$, and $E 2$ are given as $1.58 P a$, and $12.21 G P a$ respectively. The relaxation times $\tau_{1}$, and $\tau_{2}$, are given as $14.49 s$, and $1.60 s$ respectively. The Fracture Energy (FE) density for material fracture was obtained from the Superpave IDT strength test with a value of $0.68 \mathrm{~kJ} / \mathrm{m}^{3}$ at $-10^{\circ} \mathrm{C}$. The numerical simulation was performed using COMSOL Multiphysics $\AA$.

Figure 5a shows the evolution of damage parameter in the damage direction $\left(D_{3} 3\right)$ i.e. 33direction, as a function of the number of load cycles for the three different load frequencies. The result in Figure 5a shows that the material fractures when it approaches a critical damage density of 0.4 for the applied pressure. The critical damage threshold is invariant of the the rate 
of application of the repetitive load. In a similar manner, it can be observed from Figure $5 \mathrm{~b}$ that the material fracture when it approach a strain energy threshold of $0.7 \mathrm{~kJ} / \mathrm{m}^{3}$. This strain energy threshold of $0.7 \mathrm{~kJ} / \mathrm{m}^{3}$ is surprisingly close to the FE obtained from the Superpave IDT testing of the material using monotonic load. The fracture energy obtained from the Superpave IDT monotonic load condition is $0.68 \mathrm{~kJ} / \mathrm{m}^{3}$ at $-10^{\circ} \mathrm{C}$.

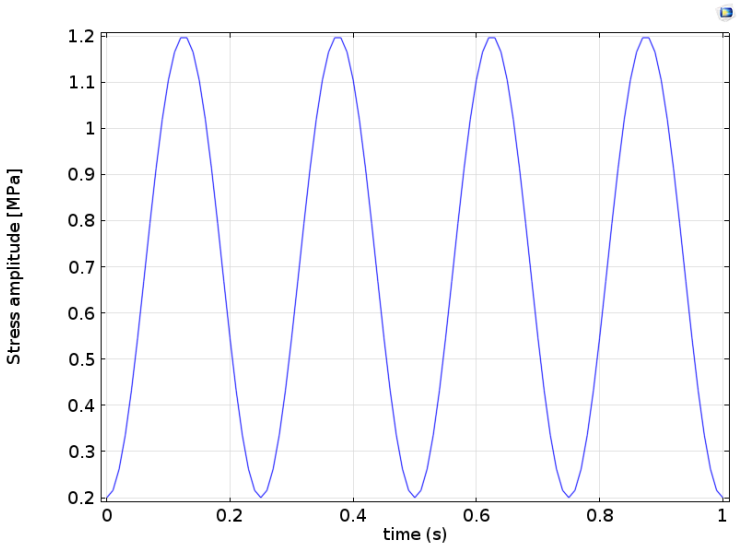

(a) Repetitive load function, $n=4$

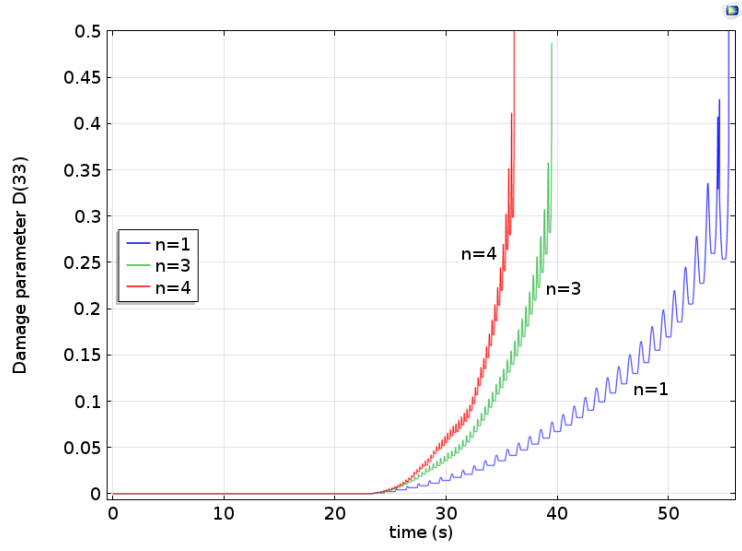

(b) Evolution of damage parameter in the damage direction $\left(D_{33}\right)$ i.e. 33-direction

Figure 4: Plot of load function for $\mathrm{n}=4$ and evolution of damage parameter $D_{33}$ with respect to time

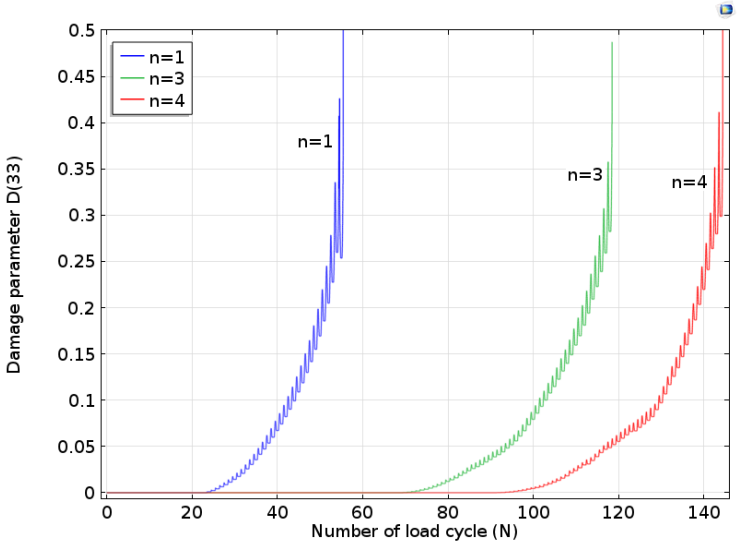

(a) Evolution of damage parameter in the damage direction $(D 33)$ i.e. 33-direction

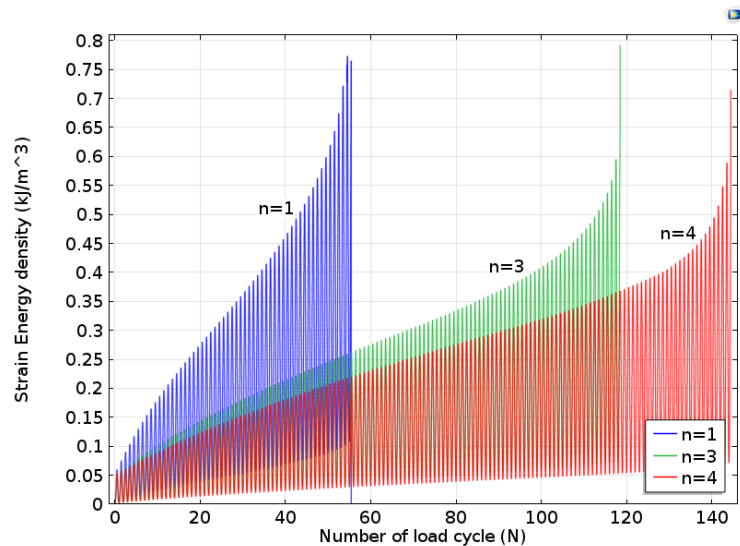

(b) strain energy density evolution at different load frequencies

Figure 5: Evolution of damage parameter $D_{33}$ and strain energy density

The result from the fatigue characterization using the level 1 energy-based damage and fracture model shows that the fundamental mechanisms of material degradation is captured by the model. Characteristic material properties such as the critical damage density and the fracture energy can be used as criteria for material fracture prediction instead of the traditional approach using the number of cycle to failure. Figure 6 shows the plot of the number of cycles to failure and the failure time at different loading frequencies. 


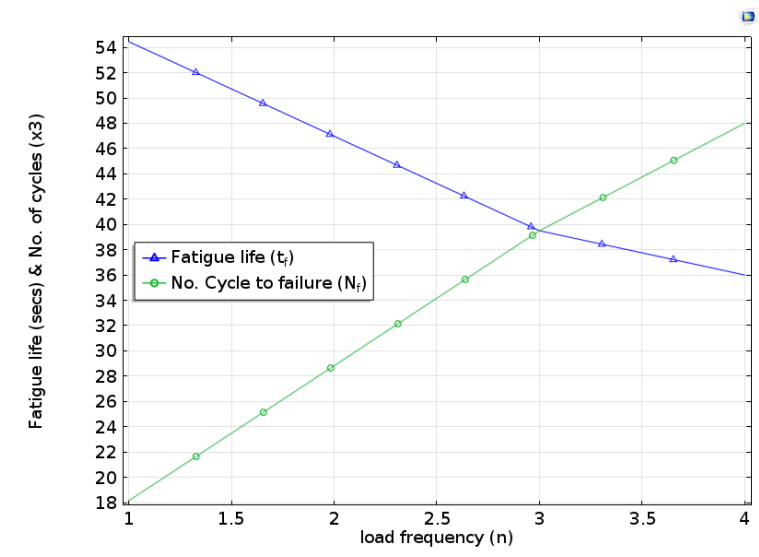

Figure 6: Plot of number of cycles to fracture and time to fracture at different loading frequencies

\section{Summary and Conclusions}

This paper presents a hierarchical approach to the evaluation of fatigue cracking in asphalt concrete pavements. The paper highlights the limitations of the existing Mechanistic Empirical (M-E) flexible pavement design guide and the importance and need for the development of mechanistic models for improved understanding of the strength and deformation mechanisms in flexible asphalt pavements.

The hierarchical approach presented in this paper provides a holistic view of the different levels of complexities in the analysis of flexible pavement performance at the material input level, the pavement response level and the damage computation or distress prediction level. The hierarchical approach also provides a systematic approach for the elimination of the empiricism associated with pavement design today and the transition towards the use of sound principles of material mechanics in pavement analysis and design.

Further validation of the Level 2 mechanics-based crack initiation prediction framework is needed to evaluate the prediction capabilities of the calibrated framework. The Level 3 energybased damage and fracture model provides a better description of the damage mechanism and the prediction of pavement response, it also exhibits the possibility of incorporating complex phenomenon such as healing into the constitutive equations in a thermodynamically consistent manner. Further development of the energy-based damage and fracture model and integration in a pavement analysis software is needed for use in the evaluation of flexible pavement performance.

\section{Acknowledgement}

The authors will like to acknowledge the support of the Swedish Transport Administration (Trafikverket) for funding this research.

\section{Bibliography}

Abu Al-Rub, R. K. and Voyiadjis, G. Z. (2003). On the coupling of anisotropic damage and plasticity models for ductile materials. International Journal of Solids and Structures, 40(11):2611-2643.

ARA Inc. (2004). Guide for mechanistic-empirical design of new and rehabilitated pavement structures. Technical report, Champaign. 
Carol, I., Rizzi, E., and Willam, K. (2001). On the formulation of anisotropic elastic degradation. i. theory based on a pseudo-logarithmic damage tensor rate. International Journal of Solids and Structures, 38(4):491-518.

Daniel, J. and Kim, Y. (2001). Laboratory Evaluation of Fatigue Damage and Healing of Asphalt Mixtures. Journal of Materials in Civil Engineering, 13(6):434-440.

Darabi, M. K., Abu Al-Rub, R. K., and Little, D. N. (2012). A continuum damage mechanics framework for modeling micro-damage healing. International Journal of Solids and Structures, 49(3-4):492-513.

Darabi, M. K., Abu Al-Rub, R. K., Masad, E. A., Huang, C.-W., and Little, D. N. (2011). A thermoviscoelastic-viscoplastic-viscodamage constitutive model for asphaltic materials. International Journal of Solids and Structures, 48(1):191-207.

Das, P. K., Birgisson, B., Jelagin, D., and Kringos, N. (2013). Investigation of the asphalt mixture morphology influence on its ageing susceptibility. Materials and Structures, 48(4):987-1000.

Dinegdae, Y. H., Onifade, I., Jelagin, D., and Birgisson, B. (2015). Mechanics-based top-down fatigue cracking initiation prediction framework for asphalt pavements. Road Materials and Pavement Design, 0(0):1-21.

Florida Department of Transportation (FDOT) (2013). Roadway designs/pavement managements/reports Reports. www.dot.state.fl.us.

Kim, Y. R., Daniel, J. S., and Wen, H. (2002). Fatigue performance evaluation of WesTrack asphalt mixtures using viscoelastic continuum damage approach. Technical report.

Kim, Y. R. and Little, D. N. (1990). One-Dimensional Constitutive Modeling of Asphalt Concrete. Journal of Engineering Mechanics, 116(4):751-772.

Kim, Y.-R., Little, D. N., and Lytton, R. L. (2003). Fatigue and Healing Characterization of Asphalt Mixtures. Journal of Materials in Civil Engineering.

Koh, C. (2009). Tensile properties of open graded friction course (OFGC) mixture to evaluate top-down cracking performance. PhD thesis, UNIVERSITY OF FLORIDA.

Lee, H., Daniel, J., and Kim, Y. (2000). Continuum Damage Mechanics-Based Fatigue Model of Asphalt Concrete. Journal of Materials in Civil Engineering, 12(2):105-112.

Lee, H. and Kim, Y. (1998). Viscoelastic Continuum Damage Model of Asphalt Concrete with Healing. Journal of Engineering Mechanics, 124(11):1224-1232.

Lira, B., Jelagin, D., and Birgisson, B. (2012). Gradation-based framework for asphalt mixture. Materials and Structures, 46(8):1401-1414.

Little, D. N. and Bhasin, A. (2007). Exploring Mechanism of Healing in Asphalt Mixtures and Quantifying its Impact. In van der Zwaag, P. S., editor, Self Healing Materials, number 100 in Springer Series in Materials Science, pages 205-218. Springer Netherlands.

Lytton, R. L., Chen, C. W., and Little, D. N. (2001). MICRODAMAGE HEALING IN ASPHALT AND ASPHALT CONCRETE, VOLUME III: A MICROMECHANICS FRACTURE AND HEALING MODEL FOR ASPHALT CONCRETE.

Onifade, I., Birgisson, B., and Balieu, R. (2015a). Energy-based damage and fracture framework for viscoelastic asphalt concrete. Engineering Fracture Mechanics, 145:67-85.

Onifade, I., Jelagin, D., Birgisson, B., and Kringos, N. (2015b). Towards Asphalt Mixture Morphology Evaluation with the Virtual Specimen Approach. International Journal on Road Materials and Pavement Design.

Roque, R., Birgisson, B., Sangpetngam, B., and Zhang, Z. (2002). Hot Mix Asphalt Fracture Mechanics: A Fundamental Crack Growth Law for Asphalt Mixtures. In Journal of the Association of Asphalt Paving Technologists, volume 71.

Roque, R., Zhang, Z., and Sankar, B. (1999). Determination of crack growth rate parameters of asphalt mixtures using the superpave IDT. Proceedings of the Association of Asphalt Paving Technologists, 68:404-433.

Schapery, R. (1990). A theory of mechanical behavior of elastic media with growing damage and other changes in structure. Journal of the Mechanics and Physics of Solids, 38(2):215-253.

Simo, J. C. and Ju, J. W. (1987a). Strain- and stress-based continuum damage models-i. formulation. International Journal of Solids and Structures, 23(7):821-840.

Simo, J. C. and Ju, J. W. (1987b). Strain- and stress-based continuum damage models-II. computational aspects. International Journal of Solids and Structures, 23(7):841-869.

Sullivan, R. W. (2008). Development of a viscoelastic continuum damage model for cyclic loading. Mechanics of Time-Dependent Materials, 12(4):329-342.

Viljoen, A. (2001). Estimating asphalt temperatures from air temperatures and basic sky parameters. Internal report Report CR-2001/78, CSIR Transportek, retoria.

Voyiadjis, G. Z., Taqieddin, Z. N., and Kattan, P. I. (2009). Theoretical formulation of a coupled elastic-plastic anisotropic damage model for concrete using the strain energy equivalence concept. International Journal of Damage Mechanics, 18(7):603-638. 
Yideti, T. F., Birgisson, B., Jelagin, D., and Guarin, A. (2014). Packing theory-based framework for evaluating resilient modulus of unbound granular materials. International Journal of Pavement Engineering, 15(8):689697.

Zhang, Z., Roque, R., Birgisson, B., and Sangpetngam, B. (2001). Identification and verification of a suitable crack growth law (with discussion). Journal of the Association of Asphalt Paving Technologists, 70. 